\title{
A Novel Pythagorean Group Decision-Making Method Based on Evidence Theory and Interactive Power Averaging Operator
}

\author{
Zhaohe Wan $\mathbb{D}^{1},{ }^{1}$ Minghua Shi $\mathbb{D},{ }^{2,3}$ Feng Yang, ${ }^{4}$ and Guang Zhu $\mathbb{D}^{1}$ \\ ${ }^{1}$ College of Economics and Management, De La Salle University, Dasmarias 4115, Philippines \\ ${ }^{2}$ College of Finance and Mathematics, West Anhui University, Lu'an 237012, China \\ ${ }^{3}$ Business School, University of Shanghai for Science and Technology, Shanghai 200093, China \\ ${ }^{4}$ College of Management, Henan University of Chinese Medicine, Zhengzhou 450046, Henan, China \\ Correspondence should be addressed to Guang Zhu; 2404440991@qq.com
}

Received 25 March 2021; Revised 23 July 2021; Accepted 11 August 2021; Published 20 August 2021

Academic Editor: Zhen Zhang

Copyright $\odot 2021$ Zhaohe Wan et al. This is an open access article distributed under the Creative Commons Attribution License, which permits unrestricted use, distribution, and reproduction in any medium, provided the original work is properly cited.

\begin{abstract}
Since Pythagorean fuzzy sets can better reflect the cognition of the decision objects for experts, researchers have begun to pay increasingly more attention to them in recent years. The majority of the research on Pythagorean fuzzy environment assumes that the decision maker is completely rational and does not consider the correlation among the attribute variables. In view of the above, this paper proposes a method to solve the multiple attribute group decision-making problem based on D-S theory and interactive power averaging operator. First, the new Pythagorean fuzzy interactive weighted power average operator is designed to aggregate the attribute evaluation information given by experts one by one, and the comprehensive evaluation information of each expert is obtained. Then, the expert comprehensive evaluation information is aggregated by the rule of evidence combination to obtain the comprehensive evidence information and confidence interval of each candidate. Then, the decision-making method for candidate alternatives is performed by the possibility discriminant rule. The design method considers not only the decision makers' bounded rationality but also the correlation among the attribute variables. Finally, the selection of the energy exploitation plan illustrates the feasibility and effectiveness of the proposed group decision method.
\end{abstract}

\section{Introduction}

Since Bellman and Zadeh formally proposed the concept of fuzzy decision in 1970, it has attracted a large number of scholars to study this field of decision-making due to its wide application [1]. With the deepening of research, traditional fuzzy sets can no longer meet the needs of modern decision science. Some extended fuzzy set concepts have been proposed, such as interval fuzzy sets, triangular fuzzy sets, trapezoidal fuzzy sets, type-2 fuzzy sets, and type-n fuzzy sets. However, these fuzzy sets have not broken through the limitations of the dichotomy and cannot represent the psychological characteristics of negation and hesitation that people show in the decision-making process. For this reason, Atanassov expands fuzzy sets and proposes the concept of intuitionistic fuzzy sets (IFS), which uses membership, nonmembership, and hesitation to represent information more delicately [2]. In view of the fact that intuitionistic fuzzy sets can flexibly represent decision-making information from multiple perspectives of membership, nonmembership, and hesitation, it has attracted the attention of many scholars and has been widely used to solve fuzzy decision problems. In 2014, Yager proposed Pythagorean fuzzy sets on the basis of intuitionistic fuzzy sets, and the constraint conditions of intuitionistic fuzzy sets were reduced such that the sum of the squares of membership and nonmembership was less than 1 [3].

After several years of development, Pythagorean fuzzy set theory and its application research have obtained some achievements [4-7]. Zhang et al. defined some similarity measures of the Pythagorean fuzzy sets and discussed their properties [8]. They also proposed a Pythagorean fuzzy decision-making method to solve the intelligent medical diagnostic problem [8]. Wan et al. further proposed a new order relation 
for elements of Pythagorean fuzzy sets and then used it to solve decision-making problems [9]. Ejegwa defined the triparametric correlation coefficients of Pythagorean fuzzy sets and verified that they can capture the three orthodox parameters of Pythagorean fuzzy sets [10]. In order to measure the closeness between Pythagorean fuzzy information units, Thao analyzed the existing defects of intuitionistic fuzzy similarity calculation methods, proposed a new similarity calculation formula to measure the Pythagorean fuzzy numbers, and used it to solve the multiattribute decision-making problem [11]. Chinnadurai et al. proposed the Pythagorean fuzzy soft sets and defined its basic operations such as addition, intersection, union, and complement. Based on this, a multiple attribute decisionmaking method using the integration operator was designed to solve the human resource management problem [12]. Zhang et al. introduced Pythagorean fuzzy preference relations and its additive consistency, which expanded the application scope of preference relations [13]. Given that the Muirhead mean operator can capture the correlation between multiple decision variables, Liu et al. discussed the relevant properties of the Muirhead mean operator in the Pythagorean fuzzy linguistic environment and proposed a Pythagorean linguistic multiple attribute decision-making method [14]. Considering the cross relationship between the degree of membership and the degree of nonmembership, Wang et al. proposed the interactive algorithm of Pythagorean fuzzy number and some collections based on interactive operations and used it to solve the multiple attribute decision-making problem of enterprise resource planning [15]. Combining the dual hesitant fuzzy sets and Pythagorean fuzzy sets, Ji et al. proposed probabilistic dual hesitant Pythagorean fuzzy sets and designed a new decisionmaking method to solve the multiple attribute decision-making problem in portfolio selection [16]. Wan et al. developed a Pythagorean fuzzy group decision method based on the degree of truth and information entropy [17]. Akram et al. proposed a novel approach based on the Hamacher operator for complex Pythagorean fuzzy decision-making [18]. Considering group utility measures and individual regret measures, Ma et al. developed a method to solve Pythagorean fuzzy multiple attribute group decision-making [19]. Wan et al. developed a novel TOPSIS method for Pythagorean fuzzy multiple attribute group decision-making [20]. Akram et al. proposed Pythagorean fuzzy Yager aggregation operators and then used them to solve the multicriteria decision-making problem [21].

However, the abovementioned Pythagorean fuzzy decision-making problems are solved under the assumption that the decision maker is completely rational, and this assumption is difficult to satisfy in actual decision-making. Evidence theory, also known as Dempster-Shafer theory, or D-S theory for short, is based on decision makers' understanding of objective things; based on the evidence and knowledge that decision makers possess, D-S theory provides uncertainty measures for fuzzy decision information. This way of portraying uncertainty is closer to human thinking habits and can reflect the bounded rational behaviour of experts in the decision-making process. This feature makes evidence theory widely used to solve fuzzy decision-making problems. However, to date, no evidence has been used to synthesize Pythagorean fuzzy information. In addition, the power average operator [22] is a classic fuzzy information gathering tool. Since it was proposed, it has received attention from many scholars and has been extended to different fuzzy environments. The common power average operators are the hesitant fuzzy power aggregation operator, intuitionistic fuzzy power average operator, and triangular intuitionistic fuzzy power average operator; they are used to solve different ambiguities in the decision-making information aggregation problem in a fuzzy environment. However, these power average operators can gather fuzzy information only from the perspective of the overall equilibrium and cannot capture the relevant information between attributes. He et al. stated that interactive computing can capture the relevant information between attributes and the information between membership and nonmembership [24]. In view of the above analysis, this paper proposes an improved power averaging operator, the Pythagorean interactive power averaging operator, combined with evidence theory, and a Pythagorean fuzzy group decision method under bounded rationality.

\section{Evidence Theory}

The recognition framework refers to a limited and complete set of all possible results of a certain proposition. The construction of the recognition framework can transform abstract propositional operations into set operations. Important concepts such as the trust function, likelihood function, and evidence combination function of D-S theory are all based on the recognition framework.

Definition 1 (see [25]). $\mathrm{F}$ is set as the DS identification framework, and $F=\left\{F_{1}, F_{2}, \ldots, F_{n}\right\}$ which is a set of mutually independent conclusions. Then, the basic probability assignment (BPA) function of the framework, also known as the mass function, is the mapping: $m: 2^{F} \longrightarrow[0,1]$, which satisfies the following conditions: $(1) m(\varnothing)=0 ; 2)$ and (2) $\sum_{A \in F} m(A)=1$. Among the components, $\varnothing$ is the empty set, $A$ is any subset of $F$, and $2^{F}=\left\{\varnothing,\left\{F_{1}\right\}, \ldots,\left\{F_{n}\right\},\left\{F_{1}, F_{2}\right\}\right.$, $\left.\ldots,\left\{F_{1}, F_{n}\right\}, \ldots, F\right\}$ is the set consisting of all subsets of $F$.

Definition 2 (see [25]). Let $F$ be a D-S recognition framework, $F=\left\{F_{1}, F_{2}, \ldots, F_{n}\right\}$ be a set of mutually independent conclusions, and $\mathrm{A}$ and $\mathrm{B}$ be subsets of $F$. Then, the belief measure (Bel) function and the plausibility measure (Pl) function are as follows:

$$
\begin{aligned}
& \operatorname{Bel}(A)=\sum_{B \subseteq A} m(B), \\
& P l(A)=\sum_{B \cap A \neq \varnothing} m(B),
\end{aligned}
$$

where $\operatorname{Bel}(A)$ represents the credibility that the evidence $A$ must occur and $\mathrm{Pl}(A)$ represents the credibility that the evidence $A$ may occur. Obviously, there is $P l(A) \geq \operatorname{Bel}(A)$ for any subset $A$ of $F$, so the support interval of $A$ can be expressed as $[\operatorname{Bel}(A), P l(A)]$, also known as the confidence interval of $A$. In order to fuse multiple pieces of evidentiary 
information to implement optimal decision-making, Dempster proposed the following orthogonal sum rule.
Theorem 1 (see [26]). Suppose that $m_{1}, m_{2}, \ldots, m_{n}$ is the mass function under the same recognition framework, and the corresponding evidence is $A_{1}, A_{2}, \ldots, A_{n}$. Then,

$$
m(A)=m_{1}\left(A_{1}\right) \oplus m_{2}\left(A_{2}\right) \oplus \cdots m_{n}\left(A_{n}\right)= \begin{cases}0, & A=\varnothing, \\ \frac{\sum_{A_{1} \cap A_{2} \cap \cdots \cap A_{n}=A} m_{1}\left(A_{1}\right) m_{2}\left(A_{2}\right) \cdots m_{n}\left(A_{n}\right)}{1-\sum_{A_{1} \cap A_{2} \cap \cdots \cap A_{n}=\varnothing} m_{1}\left(A_{1}\right) m_{2}\left(A_{2}\right) \cdots m_{n}\left(A_{n}\right)}, & A \neq \varnothing .\end{cases}
$$

\section{Pythagorean Interactive Power Average Operator}

\subsection{Pythagorean Fuzzy Sets}

Definition 3 (see [7]). Suppose that $X$ is a given universe of discourse. Then, a Pythagorean fuzzy set $A$ on $X$ is $A=\{\langle x, r(x), \mathrm{d}(x)\rangle \mid x \in X\}$, where $r(x)$ is the strength of the commitment given $x$ and $\mathrm{d}(x)$ is the bias value of the commitment, $r(x) \in[0,1]$ and $\mathrm{d}(x) \in[0,1]$.

Zhang and $\mathrm{Xu}$ further proposed the concept of a Pythagorean fuzzy number. That is, the binary array of $\alpha=P\left(\mu_{\alpha}, v_{\alpha}\right)$ is called a Pythagorean fuzzy number, where $\mu_{\alpha}$ and $v_{\alpha}$ satisfy the constraint conditions of $\left(\mu_{\alpha}\right)^{2}+\left(v_{\alpha}\right)^{2} \leq 1$. The following algorithms and binary relations are given to open the application space of Pythagorean fuzzy sets.

Definition 4 (see [8]). Let $\alpha_{i}=P\left(\mu_{\alpha_{i}}, v_{\alpha_{i}}\right)(i=1,2)$ and $\alpha=$ $P\left(\mu_{\alpha}, v_{\alpha}\right)$ be three Pythagorean fuzzy numbers. Then,
(1) $\alpha_{1} \oplus \alpha_{2}=P\left(\sqrt{\mu_{\alpha_{1}}^{2}+\mu_{\alpha_{2}}^{2}-\mu_{\alpha_{1}}^{2} \mu_{\alpha_{2}}^{2}}, v_{\alpha_{1}} v_{\alpha_{2}}\right)$
(2) $\alpha_{1} \otimes \alpha_{2}=P\left(\mu_{\alpha_{1}} \mu_{\alpha_{2}}, \sqrt{v_{\alpha_{1}}^{2}+v_{\alpha_{2}}^{2}-v_{\alpha_{1}}^{2} v_{\alpha_{2}}^{2}}\right)$
(3) $\lambda \alpha=P\left(\sqrt{1-\left(1-\mu_{\alpha}^{2}\right)^{\lambda}}, v_{\alpha}^{\lambda}\right) ; \lambda>0$
(4) $\alpha^{\lambda}=P\left(\mu_{\alpha}^{\lambda}, \sqrt{1-\left(1-v_{\alpha}^{2}\right)^{\lambda}}\right) ; \lambda>0$

However, the above algorithm does not consider the interaction between the degree of membership and the degree of nonmembership, and abnormal phenomena may occur in the calculation.

Example 1. Suppose $\alpha_{1}=P(0.6,0.4), \alpha_{2}=P(0.5,0.6)$, and $\alpha_{3}=P(0.7,0.0)$ are three Pythagorean fuzzy numbers; the corresponding weight vector is $w=\left(w_{1}, w_{2}, w_{3}\right)^{T}=(0.25,0.4,0.35)^{T}$. Then, the arithmetic weighted average of $\alpha_{i}(i=1,2,3)$ obtained by Definition 4 is

$$
\alpha=w_{1} \alpha_{1} \oplus w_{2} \alpha_{2} \oplus w_{3} \alpha_{3}=P(0.6045,0) .
$$

This means that when calculating the arithmetic weighted average of $\alpha_{i}(i=1,2,3)$, the nonmembership of $\alpha_{1}$ and $\alpha_{2}$ has no effect, which is puzzling. For this reason, Wei and others stipulated the following interactive Pythagorean fuzzy number algorithm.

Definition 5 (see [27]). Let $\alpha_{i}=P\left(\mu_{\alpha_{i}}, v_{\alpha_{i}}\right)(i=1,2)$ and $\alpha=$ $P\left(\mu_{\alpha}, v_{\alpha}\right)$ be three Pythagorean fuzzy numbers. Then,
(1) $\alpha_{1} \oplus \alpha_{2}=P\left(\sqrt{\mu_{\alpha_{1}}^{2}+\mu_{\alpha_{2}}^{2}-\mu_{\alpha_{1}}^{2} \mu_{\alpha_{2}}^{2}}\right.$, $\left.\sqrt{v_{\alpha_{1}}^{2}+v_{\alpha_{2}}^{2}-v_{\alpha_{1}}^{2} v_{\alpha_{2}}^{2}-\mu_{\alpha_{1}}^{2} v_{\alpha_{2}}^{2}-v_{\alpha_{1}}^{2} \mu_{\alpha_{2}}^{2}}\right)$

(2) $\alpha_{1} \otimes \alpha_{2}=P\left(\sqrt{\mu_{\alpha_{1}}^{2}+\mu_{\alpha_{2}}^{2}-\mu_{\alpha_{1}}^{2} \mu_{\alpha_{2}}^{2}-v_{\alpha_{1}}^{2} \mu_{\alpha_{2}}^{2}-\mu_{\alpha_{1}}^{2} v_{\alpha_{2}}^{2}}\right.$, $\left.\sqrt{v_{\alpha_{1}}^{2}+v_{\alpha_{2}}^{2}-v_{\alpha_{1}}^{2} v_{\alpha_{2}}^{2}}\right)$

(3) $\lambda \alpha=P\left(\sqrt{1-\left(1-\mu_{\alpha}^{2}\right)^{\lambda}}\right.$, $\left.\sqrt{\left(1-\mu_{\alpha}^{2}\right)^{\lambda}-\left(1-\left(\mu_{\alpha}^{2}+v_{\alpha}^{2}\right)\right)^{\lambda}}\right) ; \lambda>0$

(4) $\alpha^{\lambda}=P\left(\sqrt{\left(1-v_{\alpha}^{2}\right)^{\lambda}-\left(1-\left(\mu_{\alpha}^{2}+v_{\alpha}^{2}\right)\right)^{\lambda}}\right.$, $\left.\sqrt{1-\left(1-v_{\alpha}^{2}\right)^{\lambda}}\right) ; \lambda>0$

Using the interactive algorithm, recalculate Example 1 to obtain

$$
\alpha=w_{1} \alpha_{1} \oplus w_{2} \alpha_{2} \oplus w_{3} \alpha_{3}=P(0.6084,0.4226) .
$$

$\min \left(v_{\alpha_{1}}, v_{\alpha_{2}}, v_{\alpha_{3}}\right) \leq v_{\alpha}=0.4226 \leq \max \left(v_{\alpha_{1}}, v_{\alpha_{2}}, v_{\alpha_{3}}\right)$ shows that the interactive algorithm is closer to the actual situation.

Huang et al. stipulated the following Pythagorean fuzzy number ranking criteria and distance measure.

Definition 6 (see [30]). Set $\alpha=P\left(\mu_{\alpha}, v_{\alpha}\right)$ as a Pythagorean fuzzy number. Then, the score function of $\alpha$ is $s_{\alpha}=\left(\mu_{\alpha}\right)^{2}-\left(v_{\alpha}\right)^{2}$. For any two Pythagorean fuzzy numbers $\alpha_{1}$ and $\alpha_{2}$, the following sorting criteria exist:

(1) If $s_{\alpha_{1}}>s_{\alpha_{2}}$, then $\alpha_{1}>\alpha_{2}$

(2) If $s_{\alpha_{1}}=s_{\alpha_{2}}$, then $\alpha_{1}=\alpha_{2}$

(3) If $s_{\alpha_{1}}<s_{\alpha_{2}}$, then $\alpha_{1}<\alpha_{2}$

Definition 7 (see [30]). Let $\alpha_{i}=P\left(\mu_{\alpha_{i}}, v_{\alpha_{i}}\right)(i=1,2)$ be two Pythagorean fuzzy numbers. Then, the distance between $\alpha_{1}$ and $\alpha_{2}$ is

$$
\mathrm{d}\left(\alpha_{1}, \alpha_{2}\right)=\frac{1}{2}\left(\left|\mu_{\alpha_{1}}-\mu_{\alpha_{2}}\right|+\left|v_{\alpha_{1}}-v_{\alpha_{2}}\right|+\left|\pi_{\alpha_{1}}-\pi_{\alpha_{2}}\right|\right),
$$

where $\pi_{\alpha_{i}}=\sqrt{1-\left(\mu_{\alpha_{i}}\right)^{2}-\left(v_{\alpha_{i}}\right)^{2}}(i=1,2)$ is the hesitation of $\alpha_{i}(i=1,2)$.

3.2. Pythagorean Interactive Power Average Operator. Based on the integrity of the data, Yager proposed a power average operator that can reduce the impact of abnormal information. 
Definition 8 (see [22]). Let $a_{i}(i=1,2, \ldots, n)$ be a sequence of real numbers. If

$$
P A\left(a_{1}, a_{2}, \ldots, a_{n}\right)=\sum_{i=1}^{n} \frac{1+T\left(a_{i}\right)}{\sum_{t=1}^{n}\left(1+T\left(a_{t}\right)\right)} a_{i},
$$

then PA is called the power average operator. Among them, $T\left(a_{i}\right)=\sum_{j=1, j \neq i}^{n} \operatorname{Sup}\left(a_{i}, a_{j}\right) ; \operatorname{Sup}\left(a_{i}, a_{j}\right)$ represents the degree of support of $a_{i}$ and $a_{j}$, and it meets the following conditions:

(1) $\operatorname{Sup}\left(a_{i}, a_{j}\right) \in[0,1]$

(2) $\operatorname{Sup}\left(a_{i}, a_{j}\right)=\operatorname{Sup}\left(a_{j}, a_{i}\right)$

(3) If $\mathrm{d}\left(a_{i}, a_{j}\right) \leq \mathrm{d}\left(a_{l}, a_{k}\right)$, then $\operatorname{Sup}\left(a_{i}, a_{j}\right) \geq \operatorname{Sup}\left(a_{l}, a_{k}\right)$

However, the power average operator can only capture the balance of the attribute information, not the associated information. For this reason, this paper proposes an improved power average operator based on the Pythagorean interactive operation: the Pythagorean interactive power average operator.
Definition 9. Let $\alpha_{i}=P\left(\mu_{\alpha_{i}}, v_{\alpha_{i}}\right) \quad(i=1,2, \ldots, n)$ be a list of Pythagorean fuzzy numbers with weights of $w_{i}(i=1,2, \ldots, n)$, where $w_{i} \in[0,1]$ and $\sum_{i=1}^{n} w_{i}=1$. Then, the Pythagorean fuzzy interactive weighted power average operator is PFIWPA:

$$
\operatorname{PFIWPA}\left(\alpha_{1}, \alpha_{2}, \ldots, \alpha_{n}\right)=\bigoplus_{i=1}^{n} \frac{w_{i}\left(1+T\left(\alpha_{i}\right)\right)}{\sum_{t=1}^{n} w_{t}\left(1+T\left(\alpha_{t}\right)\right)} \alpha_{i},
$$

where $T\left(\alpha_{i}\right)=\sum_{j=1, j \neq i}^{n} \operatorname{Sup}\left(\alpha_{i}, \alpha_{j}\right)$ and $\operatorname{Sup}\left(\alpha_{i}, \alpha_{j}\right)$ is the degree of support of $\alpha_{i}$ and $\alpha_{j}$, which satisfies the constraints:

(1) $\operatorname{Sup}\left(\alpha_{i}, \alpha_{j}\right) \in[0,1]$

(2) $\operatorname{Sup}\left(\alpha_{i}, \alpha_{j}\right)=\operatorname{Sup}\left(\alpha_{j}, \alpha_{i}\right)$

(3) If $\mathrm{d}\left(\alpha_{i}, \alpha_{j}\right) \leq \mathrm{d}\left(\alpha_{l}, \alpha_{k}\right)$, then $\operatorname{Sup}\left(\alpha_{i}, \alpha_{j}\right) \geq \operatorname{Sup}\left(\alpha_{l}, \alpha_{k}\right)$

Theorem 2. Let $\alpha_{i}=P\left(\mu_{\alpha_{i}}, v_{\alpha_{i}}\right)(i=1,2, \ldots, n)$ be a list of Pythagorean fuzzy numbers with weights of $w_{i}(i=1,2, \ldots, n)$, where $w_{i} \in[0,1]$ and $\sum_{i=1}^{n} w_{i}=1$. Then, the aggregation value obtained by the PFIWPA operator is also a Pythagorean fuzzy number, and

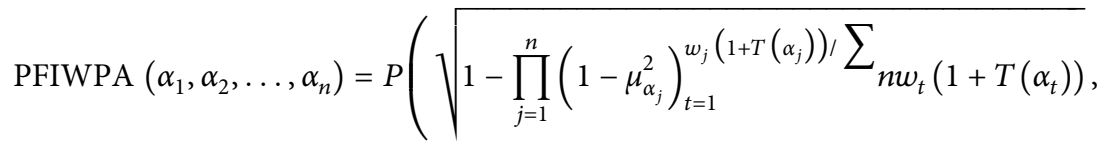

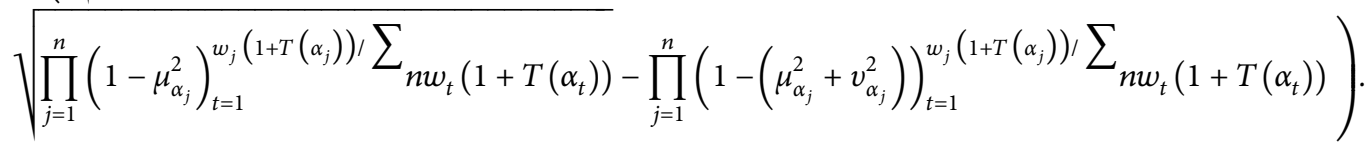

Proof. (1) When $\quad\left(\alpha_{1}, \alpha_{2}\right)=\oplus_{i=1}^{2}\left(w_{i}\left(1+\quad T\left(\alpha_{i}\right)\right) / \sum_{t=1}^{2}\right.$

$$
\begin{gathered}
\left.w_{t}\left(1+T\left(\alpha_{t}\right)\right)\right) \alpha_{i}, \\
\operatorname{PFIWPA}\left(\alpha_{1}, \alpha_{2}\right)=\stackrel{\oplus}{\oplus}_{i=1}^{2} \frac{w_{i}\left(1+T\left(\alpha_{i}\right)\right)}{\sum_{t=1}^{2} w_{t}\left(1+T\left(\alpha_{t}\right)\right)} \alpha_{i} .
\end{gathered}
$$

$$
\begin{aligned}
\frac{w_{1}\left(1+T\left(\alpha_{1}\right)\right)}{\sum_{t=1}^{2} w_{t}\left(1+T\left(\alpha_{t}\right)\right)} \alpha_{1}= & P\left(\sqrt{1-\left(1-\mu_{\alpha_{1}}^{2}\right)^{w_{1}\left(1+T\left(\alpha_{1}\right)\right) / \sum_{t=1}^{2} w_{t}\left(1+T\left(\alpha_{t}\right)\right)}},\right. \\
& \sqrt{\left.\left(1-\mu_{\alpha_{1}}^{2}\right)^{w_{1}\left(1+T\left(\alpha_{1}\right)\right) / \sum_{t=1}^{2} w_{t}\left(1+T\left(\alpha_{t}\right)\right)}-\left(1-\left(\mu_{\alpha_{1}}^{2}+v_{\alpha_{1}}^{2}\right)\right)^{w_{1}\left(1+T\left(\alpha_{1}\right)\right) / \sum_{t=1}^{2} w_{t}\left(1+T\left(\alpha_{t}\right)\right)}\right)},
\end{aligned}
$$$$
\frac{w_{2}\left(1+T\left(\alpha_{2}\right)\right)}{\sum_{t=1}^{2} w_{t}\left(1+T\left(\alpha_{t}\right)\right)} \alpha_{2}=P\left(\sqrt{1-\left(1-\mu_{\alpha_{2}}^{2}\right)^{w_{2}\left(1+T\left(\alpha_{2}\right)\right) / \sum_{t=1}^{2} w_{t}\left(1+T\left(\alpha_{t}\right)\right)}}\right.
$$

$$
\sqrt{\left(1-\mu_{\alpha_{2}}^{2}\right)^{w_{2}\left(1+T\left(\alpha_{2}\right)\right) / \sum_{t=1}^{2} w_{t}\left(1+T\left(\alpha_{t}\right)\right)}-\left(1-\left(\mu_{\alpha_{2}}^{2}+v_{\alpha_{2}}^{2}\right)\right)^{w_{2}\left(1+T\left(\alpha_{2}\right)\right) / \sum_{t=1}^{2} w_{t}\left(1+T\left(\alpha_{t}\right)\right)}}
$$


Complexity

5

There is

$$
\begin{aligned}
& \operatorname{PFIWPA}\left(\alpha_{1}, \alpha_{2}\right)=\underset{i=1}{\oplus} \frac{w_{i}\left(1+T\left(\alpha_{i}\right)\right)}{\sum_{t=1}^{2} w_{t}\left(1+T\left(\alpha_{t}\right)\right)} \alpha_{i}
\end{aligned}
$$

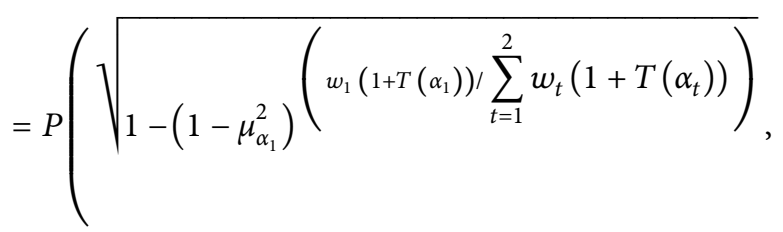

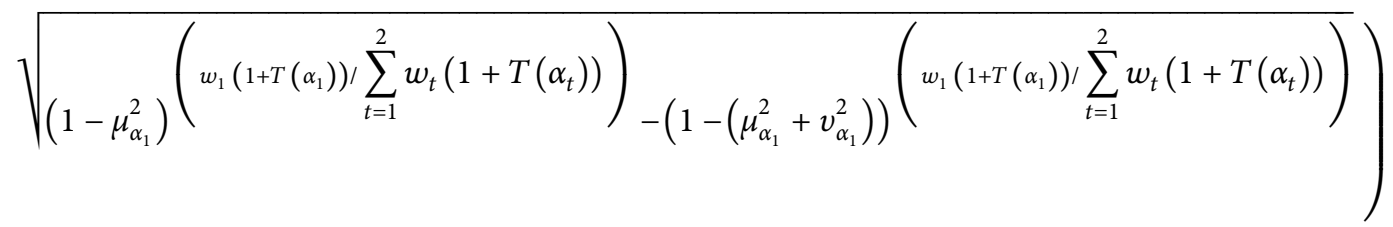

$$
\begin{aligned}
& =P\left(\sqrt{1-\left(1-\mu_{\alpha_{1}}^{2}\right)^{w_{1}\left(1+T\left(\alpha_{1}\right)\right) / \sum_{t=1}^{2} w_{t}\left(1+T\left(\alpha_{t}\right)\right)}}\right.
\end{aligned}
$$

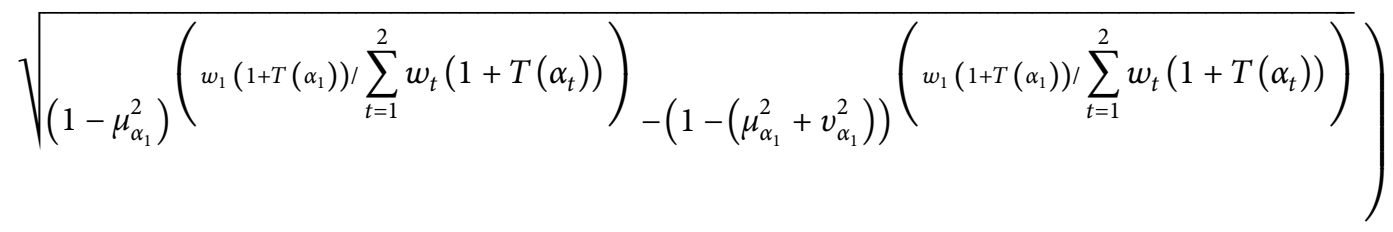

$$
\begin{aligned}
& \oplus P \sqrt{1-\left(1-\mu_{\alpha_{2}}^{2}\right)^{w_{2}\left(1+T\left(\alpha_{2}\right)\right) / \sum_{t=1}^{2} w_{t}\left(1+T\left(\alpha_{t}\right)\right)}} \\
& \sqrt{\left(1-\mu_{\alpha_{2}}^{2}\right)^{w_{2}\left(1+T\left(\alpha_{2}\right)\right) / \sum_{t=1}^{2} w_{t}\left(1+T\left(\alpha_{t}\right)\right)}-\left(1-\left(\mu_{\alpha_{2}}^{2}+v_{\alpha_{2}}^{2}\right)\right)^{w_{2}\left(1+T\left(\alpha_{2}\right)\right) / \sum_{t=1}^{2} w_{t}\left(1+T\left(\alpha_{t}\right)\right)}} \\
& =P\left(\sqrt{1-\left(1-\mu_{\alpha_{1}}^{2}\right)^{w_{1}\left(1+T\left(\alpha_{1}\right)\right) / \sum_{t=1}^{2} w_{t}\left(1+T\left(\alpha_{t}\right)\right)}\left(1-\mu_{\alpha_{2}}^{2}\right)^{w_{2}\left(1+T\left(\alpha_{2}\right)\right) / \sum_{t=1}^{2} w_{t}\left(1+T\left(\alpha_{t}\right)\right)}}\right. \\
& \sqrt{1-\left(1-\mu_{\alpha_{1}}^{2}\right)^{w_{1}\left(1+T\left(\alpha_{1}\right)\right) / \sum_{t=1}^{2} w_{t}\left(1+T\left(\alpha_{t}\right)\right)}\left(1-\mu_{\alpha_{2}}^{2}\right) w_{2}^{w_{2}\left(1+T\left(\alpha_{2}\right)\right) / \sum_{t=1}^{2} w_{t}\left(1+T\left(\alpha_{t}\right)\right)}-\left(1-\left(\mu_{\alpha_{1}}^{2}+v_{\alpha_{1}}^{2}\right)\right)^{w_{1}\left(1+T\left(\alpha_{1}\right)\right) / \sum_{t=1}^{2} w_{t}\left(1+T\left(\alpha_{t}\right)\right)}\left(1-\left(\mu_{\alpha_{2}}^{2}+v_{\alpha_{2}}^{2}\right)\right)^{w_{2}\left(1+T\left(\alpha_{2}\right)\right) / \sum_{t=1}^{2} w_{t}\left(1+T\left(\alpha_{t}\right)\right)}}
\end{aligned}
$$




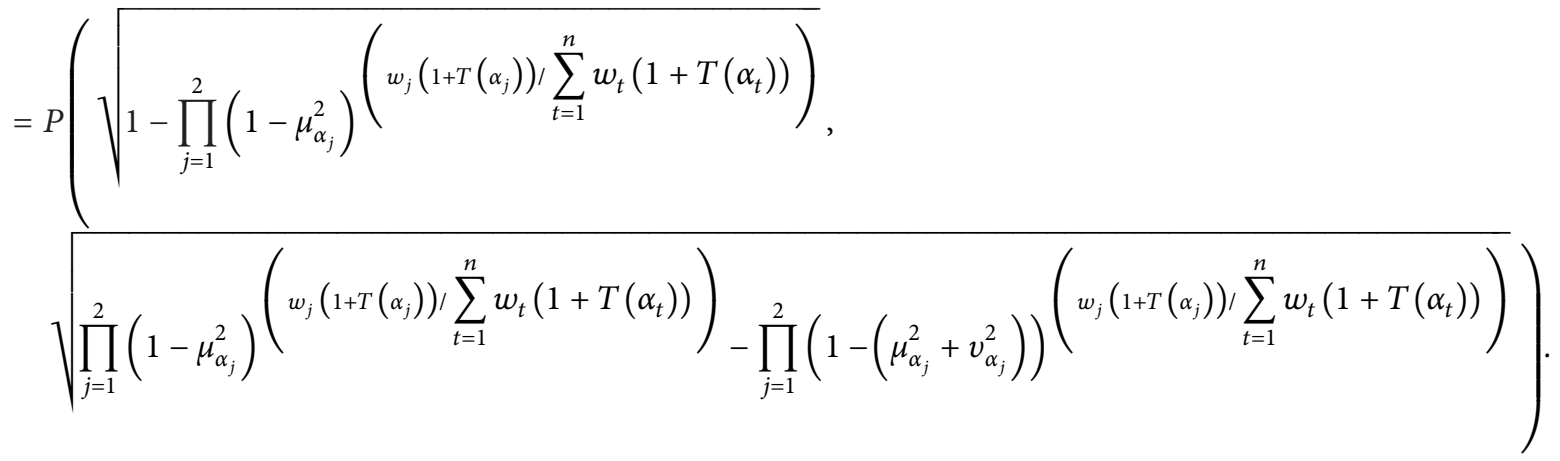

Therefore, when $\left(\alpha_{1}, \alpha_{2}, \ldots, \alpha_{k}\right) \oplus_{i=1}^{k}\left(w_{i}(1+T\right.$ $\left.\left.\left(\alpha_{i}\right)\right) / \sum_{t=} 1^{n} w_{t}\left(1+T\left(\alpha_{t}\right)\right)\right) \alpha_{i}$, formula $(8)$ is established.

(2) Assuming $\left(\alpha_{1}, \alpha_{2}, \ldots, \alpha_{k}\right)=\oplus_{i=1}^{k}\left(w_{i}\left(1+T\left(\alpha_{i}\right)\right) /\right.$ $\left.\sum_{t=1}^{n} w_{t}\left(1+T\left(\alpha_{t}\right)\right)\right) \alpha_{i} \quad\left(\left(\alpha_{1}, \alpha_{2}, \ldots, \alpha_{k}\right)=\oplus_{i=1}^{k}\left(w_{i}\right.\right.$
$\left.\left.\left(1+T\left(\alpha_{i}\right)\right) / \sum_{t=1}^{n} w_{t}\left(1+T\left(\alpha_{t}\right)\right)\right) \alpha_{i}\right)$,formula (8) is established; considering $\left(\alpha_{1}, \alpha_{2}, \ldots, \alpha_{k}\right)=\oplus_{i=1}^{k}$ $\left(w_{i}\left(1+T\left(\alpha_{i}\right)\right) / \sum_{t=1}^{n} w_{t}\left(1+T\left(\alpha_{t}\right)\right)\right) \alpha_{i}$, formula $(8)$ is still established:

$\operatorname{PFIWPA}\left(\alpha_{1}, \alpha_{2}, \ldots, \alpha_{k}\right)=\underset{i=1}{\stackrel{\oplus}{\oplus}} \frac{w_{i}\left(1+T\left(\alpha_{i}\right)\right)}{\sum_{t=1}^{n} w_{t}\left(1+T\left(\alpha_{t}\right)\right)} \alpha_{i}$

$$
\begin{aligned}
& =P\left(\sqrt{1-\prod_{j=1}^{k}\left(1-\mu_{\alpha_{j}}^{2}\right)^{w_{j}\left(1+T\left(\alpha_{j}\right)\right) / \sum_{t=1}^{n} w_{t}\left(1+T\left(\alpha_{t}\right)\right)},}\right. \\
& \left.\sqrt{\prod_{j=1}^{k}\left(1-\mu_{\alpha_{j}}^{2}\right)^{w_{j}\left(1+T\left(\alpha_{j}\right)\right) / \sum_{t=1}^{n} w_{t}\left(1+T\left(\alpha_{t}\right)\right)}-\prod_{j=1}^{k}\left(1-\left(\mu_{\alpha_{j}}^{2}+v_{\alpha_{j}}^{2}\right)\right)^{w_{j}\left(1+T\left(\alpha_{j}\right)\right) / \sum_{t=1}^{n} w_{t}\left(1+T\left(\alpha_{t}\right)\right)}}\right) .
\end{aligned}
$$

Then,

$\operatorname{PFIWPA}\left(\alpha_{1}, \alpha_{2}, \ldots, \alpha_{k+1}\right)=\stackrel{\oplus}{i=1}_{i+1}^{k} \frac{w_{i}\left(1+T\left(\alpha_{i}\right)\right)}{\sum_{t=1}^{n} w_{t}\left(1+T\left(\alpha_{t}\right)\right)} \alpha_{i}=\stackrel{\oplus}{\oplus} \frac{w_{i}\left(1+T\left(\alpha_{i}\right)\right)}{\sum_{t=1}^{n} w_{t}\left(1+T\left(\alpha_{t}\right)\right)} \alpha_{i} \oplus \frac{w_{i}\left(1+T\left(\alpha_{i}\right)\right)}{\sum_{t=1}^{n} w_{t}\left(1+T\left(\alpha_{t}\right)\right)} \alpha_{k+1}$ 
Complexity

7

$$
\begin{aligned}
& =P\left(\sqrt{1-\prod_{j=1}^{k}\left(1-\mu_{\alpha_{j}}^{2}\right)^{w_{j}\left(1+T\left(\alpha_{j}\right)\right) / \sum_{t=1}^{n} w_{t}\left(1+T\left(\alpha_{t}\right)\right)}},\right. \\
& \sqrt{\prod_{j=1}^{k}\left(1-\mu_{\alpha_{j}}^{2}\right)^{w_{j}\left(1+T\left(\alpha_{j}\right)\right) / \sum_{t=1}^{n} w_{t}\left(1+T\left(\alpha_{t}\right)\right)}-\prod_{j=1}^{k}\left(1-\left(\mu_{\alpha_{j}}^{2}+v_{\alpha_{j}}^{2}\right)\right)^{w_{j}\left(1+T\left(\alpha_{j}\right)\right) / \sum_{t=1}^{n} w_{t}\left(1+T\left(\alpha_{t}\right)\right)}} \\
& \oplus P\left(\sqrt{1-\left(1-\mu_{\alpha_{k+1}}^{2}\right)^{w_{k+1}\left(1+T\left(\alpha_{k+1}\right)\right) / \sum_{t=1}^{k+1} w_{t}\left(1+T\left(\alpha_{t}\right)\right)},}\right. \\
& \left.\sqrt{\left(1-\mu_{\alpha_{k+1}}^{2}\right)^{w_{k+1}\left(1+T\left(\alpha_{k+1}\right)\right) / \sum_{t=1}^{k+1} w_{t}\left(1+T\left(\alpha_{t}\right)\right)}-\left(1-\left(\mu_{\alpha_{k+1}}^{2}+v_{\alpha_{k+1}}^{2}\right)\right)^{w_{k+1}\left(1+T\left(\alpha_{k+1}\right)\right) / \sum_{t=1}^{k+1} w_{t}\left(1+T\left(\alpha_{t}\right)\right)}}\right) \\
& =P\left(\sqrt{1-\prod_{j=1}^{k+1}\left(1-\mu_{\alpha_{j}}^{2}\right)^{w_{j}\left(1+T\left(\alpha_{j}\right)\right) / \sum_{t=1}^{n} w_{t}\left(1+T\left(\alpha_{t}\right)\right)}},\right. \\
& \sqrt{\prod_{j=1}^{k+1}\left(1-\mu_{\alpha_{j}}^{2}\right)^{w_{j}\left(1+T\left(\alpha_{j}\right)\right) / \sum_{t=1}^{n} w_{t}\left(1+T\left(\alpha_{t}\right)\right)}-\prod_{j=1}^{k+1}\left(1-\left(\mu_{\alpha_{j}}^{2}+v_{\alpha_{j}}^{2}\right)\right)^{w_{j}\left(1+T\left(\alpha_{j}\right)\right) / \sum_{t=1}^{n} w_{t}\left(1+T\left(\alpha_{t}\right)\right)}} .
\end{aligned}
$$

Therefore, when $n \geq 2$, formula (8) is also established. In summary, formula $(8)$ is established when $(n \geq 2)$ from the mathematical induction; obviously, the aggregation value of the PFIWPA operator is still a Pythagorean fuzzy number.

Note that if $T\left(\alpha_{i}\right)=c(i=1,2, \ldots, n)$, then

$$
\begin{aligned}
& \text { PFIWPA }\left(\alpha_{1}, \alpha_{2}, \ldots, \alpha_{n}\right) \\
& =P\left(\sqrt{1-\prod_{j=1}^{n}\left(1-\mu_{\alpha_{j}}^{2}\right)\left(w_{j}\left(1+T\left(\alpha_{j}\right)\right) / \sum_{t=1}^{n} w_{t}\left(1+T\left(\alpha_{t}\right)\right)\right)},\right.
\end{aligned}
$$

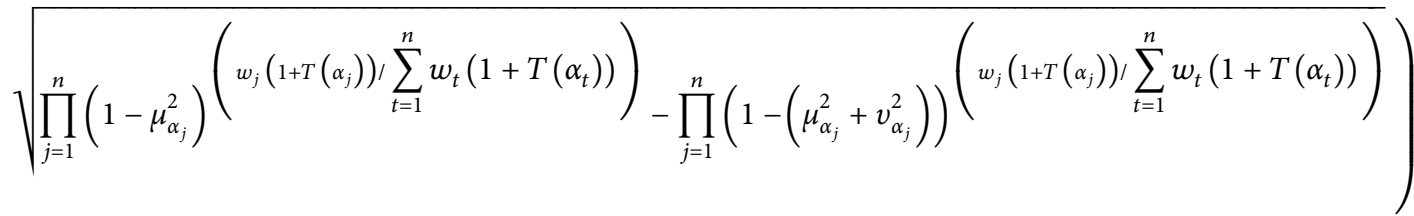

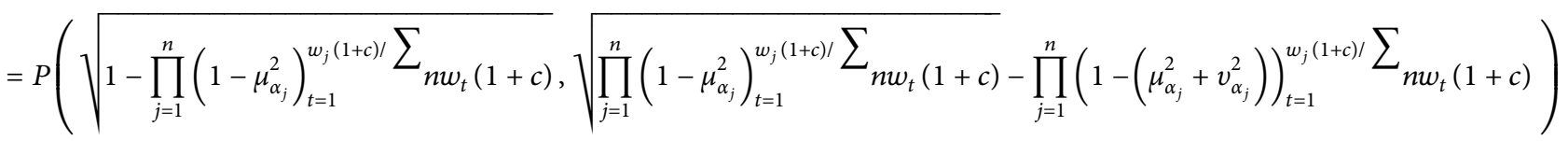

$$
\begin{aligned}
& =P\left(\sqrt{1-\prod_{j=1}^{n}\left(1-\mu_{\alpha_{j}}^{2}\right)^{w_{j}}}, \sqrt{\prod_{j=1}^{n}\left(1-\mu_{\alpha_{j}}^{2}\right)^{w_{j}}-\prod_{j=1}^{n}\left(1-\left(\mu_{\alpha_{j}}^{2}+v_{\alpha_{j}}^{2}\right)\right)^{w_{j}}}\right) \\
& =\operatorname{PFIWA}\left(\alpha_{1}, \alpha_{2}, \ldots, \alpha_{n}\right) \text {. }
\end{aligned}
$$


That is, the PFIWPA operator degenerates into the Pythagorean fuzzy interactive weighted average (PFIWA) operator.
Theorem 3. Let $\alpha_{i}=P\left(\mu_{\alpha_{i}}, v_{\alpha_{i}}\right)$ be a list of Pythagorean fuzzy numbers. If $\alpha_{i}=\alpha=P\left(\mu_{\alpha}, v_{\alpha}\right) \quad(i=1,2, \ldots, n)$, then PFIWPA $\left(\alpha_{1}, \alpha_{2}, \ldots, \alpha_{n}\right)=\alpha$

Proof.

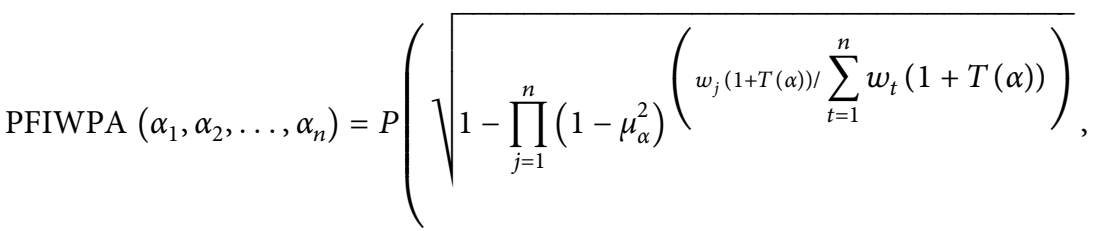

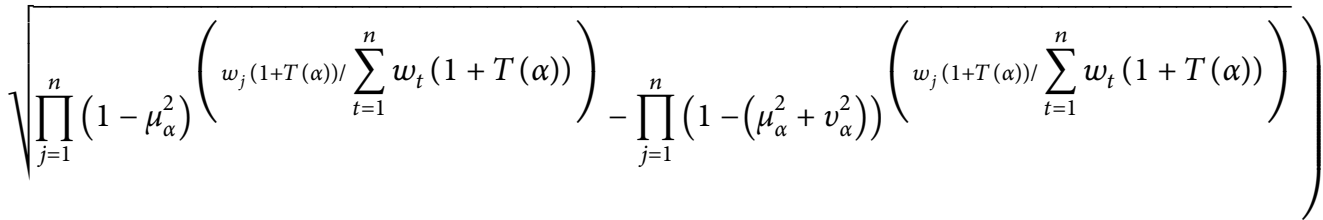

$$
\begin{aligned}
& =P\left(\sqrt{1-\prod_{j=1}^{n}\left(1-\mu_{\alpha}^{2}\right)^{w_{j}}}, \sqrt{\prod_{j=1}^{n}\left(1-\mu_{\alpha}^{2}\right)^{w_{j}}-\prod_{j=1}^{n}\left(1-\left(\mu_{\alpha}^{2}+v_{\alpha}^{2}\right)\right)^{w_{j}}}\right) \\
& =P\left(\sqrt{1-\left(1-\mu_{\alpha}^{2}\right) \sum_{j=1} n w_{j}}, \sqrt{\left(1-\mu_{\alpha}^{2}\right) \sum_{j=1} n w_{j}}-\left(1-\left(\mu_{\alpha}^{2}+v_{\alpha}^{2}\right)\right) \sum_{j=1} n w_{j}\right) \\
& =P\left(\sqrt{1-\left(1-\mu_{\alpha}^{2}\right)}, \sqrt{\left(1-\mu_{\alpha}^{2}\right)-\left(1-\left(\mu_{\alpha}^{2}+v_{\alpha}^{2}\right)\right)}\right) .
\end{aligned}
$$

Theorem 4. Let $\alpha_{i}, \alpha_{i}^{\prime}(i=1,2, \ldots, n)$ can be two columns of Proof. Pythagorean fuzzy numbers.

If $\alpha_{1}^{\prime}, \alpha_{2}^{\prime}, \ldots, \alpha_{n}^{\prime}$ is any permutation of $\alpha_{1}, \alpha_{2}, \ldots, \alpha_{n}$, then PFIWPA $\left(\alpha_{1}, \alpha_{2}, \ldots, \alpha_{n}\right)=$ PFIWPA $\left(\alpha_{1}^{\prime}, \alpha_{2}^{\prime}, \ldots, \alpha_{n}^{\prime}\right)$.

$$
\begin{aligned}
& \text { PFIWPA }\left(\alpha_{1}, \alpha_{2}, \ldots, \alpha_{n}\right)
\end{aligned}
$$

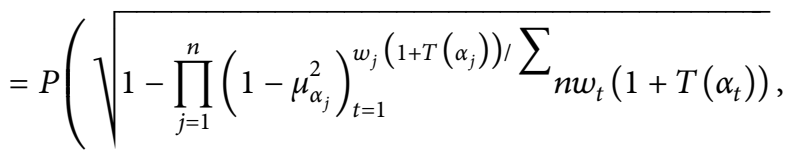

$$
\begin{aligned}
& \left.\sqrt{\prod_{j=1}^{n}\left(1-\mu_{\alpha_{j}}^{2}\right)_{t=1}^{w_{j}\left(1+T\left(\alpha_{j}\right)\right) / \sum} n w_{t}\left(1+T\left(\alpha_{t}\right)\right)}-\prod_{j=1}^{n}\left(1-\left(\mu_{\alpha_{j}}^{2}+v_{\alpha_{j}}^{2}\right)\right)_{t=1}^{w_{j}\left(1+T\left(\alpha_{j}\right)\right) / \sum} n w_{t}\left(1+T\left(\alpha_{t}\right)\right)\right)
\end{aligned}
$$

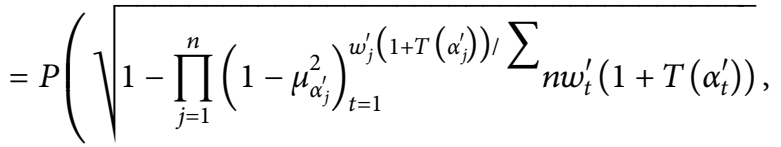

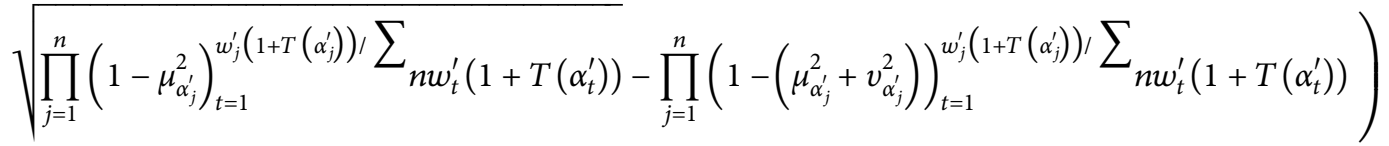

$$
\begin{aligned}
& =\operatorname{PFIWPA}\left(\alpha_{1}^{\prime}, \alpha_{2}^{\prime}, \ldots, \alpha_{n}^{\prime}\right) \text {. }
\end{aligned}
$$




\section{Pythagorean Fuzzy Multiple Attribute Group Decision-Making Method}

The problem of the multiple attribute group method in Pythagorean fuzzy environment can be described as follows: the existing decision plan set $Y=\left\{Y_{1}, Y_{2}, \ldots, Y_{n}\right\}$, $C=\left\{c_{1}, c_{2}, \ldots, c_{m}\right\}$ is the $m$ attributes of the plan, the attribute weight is $w=\left\{w_{1}, w_{2}, \ldots, w_{m}\right\},\left\{D_{1}, D_{2}, \ldots, D_{l}\right\}$ is a group of decision makers, the weight vector is $\lambda=\left(\lambda_{1}, \lambda_{2}, \ldots, \lambda_{m}\right)$, and $h_{i j}^{(k)}$ represents the Pythagorean fuzzy evaluation value given by the $k$ th decision expert $D_{k}$ to the attribute $c_{j}$ of the scheme $Y_{i}$. The method matrix provided by the decision expert $D_{k}$ can be expressed as $H^{(k)}=\left(\alpha_{i j}^{(k)}\right)_{n \times m}$. The steps of the group decision-making method of the interactive power average operator are as follows:

Step 1: convert the Pythagorean fuzzy decision matrix $H^{(k)}$ into a canonical matrix $\bar{H}^{(k)}=\left(\bar{\alpha}_{i j}^{(k)}\right)_{n \times m}$, where $\bar{\alpha}_{i j}^{(k)}=\left\{\alpha_{i j}^{(k)} \quad g_{j}:\right.$ benefit attribute $N\left(\alpha_{i j}^{(k)}\right)=P \quad\left(v_{\alpha_{i j}^{(k)}}\right.$, $\left.\mu_{\alpha_{i j}(k)}\right) g_{j}:$ cost attribute.

Step 2: calculate the degree of support $\operatorname{Sup}\left(\bar{\alpha}_{l i}^{(k)}, \bar{\alpha}_{l j}^{(k)}\right)=1-\mathrm{d}\left(\bar{\alpha}_{l i}^{(k)}, \bar{\alpha}_{l j}^{(k)}\right), \quad i, j=1,2, \ldots, m$; $l=1,2, \ldots, n, \mathrm{~d}\left(\bar{\alpha}_{l i}^{(k)}, \bar{\alpha}_{l j}^{(k)}\right)=1 / 2\left(\left|\mu_{\bar{\alpha}_{l i}^{(k)}}-\mu_{\bar{\alpha}_{l j}^{(k)}}\right|+\mid v_{\bar{\alpha}_{l i}^{(k)}}-\right.$ $\left.v_{\bar{\alpha}_{l j}^{(k)}}|+| \pi_{\bar{\alpha}_{l i}^{(k)}}-\pi_{\bar{\alpha}_{l j}^{(k)}} \mid\right)$ between variables.

Step 3: calculate the support $T\left(\bar{\alpha}_{l i}^{(k)}\right)=$ $T\left(\bar{\alpha}_{l i}^{(k)}\right)=\sum_{j=1, j \neq i}^{m} w_{j} \operatorname{Sup}\left(\bar{\alpha}_{l i}^{(k)}, \bar{\alpha}_{l j}^{(k)}\right)$ of the variable and the remaining variables and the support indices $\eta_{l i}^{(k)}=w_{i}\left(1+T\left(\bar{\alpha}_{l i}^{(k)}\right)\right) / w_{t}\left(1+T\left(\bar{\alpha}_{l t}^{(k)}\right)\right), i=1,2, \ldots, m$ and $l=1,2, \ldots, n$ of the variable.

Step 4: use the PFIWPA operator to gather the attribute values $\bar{\alpha}_{i j}^{(k)}, j=1,2, \ldots, m$, of the candidate solutions to obtain the comprehensive attribute values of the experts $D_{k}$ about the candidate solutions $x_{i}$ :

$$
\begin{aligned}
& \alpha_{i}^{k}=\operatorname{PFIWPA}\left(\bar{\alpha}_{i 1}^{(k)}, \bar{\alpha}_{i 2}^{(k)}, \ldots, \bar{\alpha}_{i m}^{(k)}\right), i=1,2, \ldots, n
\end{aligned}
$$

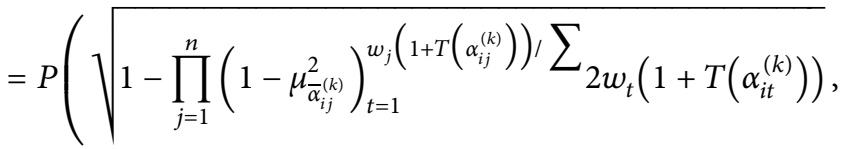

$$
\begin{aligned}
& \sqrt{\prod_{j=1}^{n}\left(1-\mu_{\bar{\alpha}_{i j}^{(k)}}^{2}\right)_{t=1}^{w_{j}\left(1+T\left(\alpha_{i j}^{(k)}\right)\right) / \sum} 2 w_{t}\left(1+T\left(\alpha_{i t}^{(k)}\right)\right)}-\prod_{j=1}^{n}\left(1-\left(\mu_{\bar{\alpha}_{i j}^{(k)}}^{2}+v_{\bar{\alpha}_{i j}^{(k)}}^{2}\right)\right)_{t=1}^{\left.w_{j}\left(1+T\left(\alpha_{i j}^{(k)}\right)\right) / \sum 2 w_{t}\left(1+T\left(\alpha_{i t}^{(k)}\right)\right)\right) .}
\end{aligned}
$$

Step 5: for each expert's standardized comprehensive evaluation information on the plan, use the evidence synthesis method to gather the results.
First, standardize the comprehensive evaluation information $\alpha_{i}^{(k)}$ solved in step 3 to obtain standardized comprehensive evaluation information $\beta_{i}^{(k)}$ :

$$
\beta_{i}^{(k)}=P\left(\mu_{\beta_{i}^{(k)}}, v_{\beta_{i}^{(k)}}\right)=\lambda_{k} P\left(\mu_{\alpha_{i}^{(k)}}, v_{\alpha_{i}^{(k)}}\right), \quad i=1,2, \ldots, n, k=1,2, \ldots, l
$$

Regarding the comprehensive evaluation information $\beta_{i}^{(k)}$ of $Y_{i}$ about the expert $D_{k}$ as a special piece of evidence, $\beta_{i}^{(k)}=P\left(\mu_{\beta_{i}^{(k)}}, v_{\beta_{i}^{(k)}}\right)$ contains three conclusions, namely, the degree of satisfaction, the degree of dissatisfaction, and the degree of uncertainty of $Y_{i}$. If $A$ indicates the degree of satisfaction of $D_{k}$ regarding $Y_{i}$, then $m_{k i}(A)=\mu_{\beta^{(k)}} / r$; If $R$ represents the degree of dissatisfaction of $D_{k}$ regarding $Y_{i}$, then $m_{k i}(R)=v_{\beta_{i}^{(k)}} / r$; If
$(A, R)$ represents the degree of uncertainty of $D_{k}$ regarding $Y_{i}$, then $m_{k i}(A, R)=\sqrt{1-\mu_{\beta_{i}^{(k)}}^{2}-v_{\beta_{i}^{(k)}}^{2}} / r$, where $r=\sqrt{1-\mu_{\beta_{i}^{(k)}}^{2}-v_{\beta_{i}^{(k)}}^{2}}+v_{\beta_{i}^{(k)}}+\mu_{\beta_{i}^{(k)}}$. Evidence synthesis rules are used to calculate the comprehensive evidence $M_{i}$ of the plan $Y_{i}$ :

$$
M_{i}(A)=m_{1 i}\left(A_{1}\right) \oplus m_{2 i}\left(A_{2}\right) \oplus \cdots \oplus m_{l i}\left(A_{l}\right)=\frac{\sum_{A_{1} \cap A_{2} \cap \cdots \cap A_{l}=A} m_{1 i}\left(A_{1}\right) m_{2 i}\left(A_{2}\right) \ldots m_{l i}\left(A_{l}\right)}{1-\sum_{A_{1} \cap A_{2} \cap \cdots \cap A_{l}=\varnothing} m_{1 i}\left(A_{1}\right) m_{2 i}\left(A_{2}\right) \ldots m_{l i}\left(A_{l}\right)}
$$

where $A, A_{1}, A_{2}, \ldots, A_{l} \in\{A, R,(A, R)\}$. 
Step 6: give the confidence interval $I_{i}$ of the candidate plan $Y_{i}$ regarding the comprehensive evidence $M_{i}=P\left(\mu_{M_{i}}, v_{M_{i}}\right), \quad$ i.e., $\quad I_{i}=\operatorname{Bel}\left[\left(x_{i}\right)=\mu_{M_{i}}\left(x_{i}\right)=\right.$ $\left.\mu_{M_{i}}+\sqrt{1-\mu_{M_{i}}^{2}-v_{M_{i}}^{2}}\right]$. Then, give the probability matrix $P=\left(p_{i j}\right)_{n \times n}$ between the candidate plans according to the confidence interval, where $p_{i j}$ is the probability that the candidate plan $Y_{i}$ is superior to $Y_{j}$. The calculation formula is $p_{i j}=\min \left\{\left[P l\left(x_{i}\right)-\right.\right.$ $\left.\operatorname{Bel}\left(x_{i}\right)\right]+\left[P l\left(x_{j}\right)-\operatorname{Bel}\left(x_{j}\right)\right], \max \left(P l\left(x_{i}\right)-\operatorname{Bel}\left(x_{j}\right)\right.$, $0)\} /\left(\left[P l\left(x_{i}\right)-\operatorname{Bel}\left(x_{i}\right)\right]+\left[\operatorname{Pl}\left(x_{j}\right)-\operatorname{Bel}\left(x_{j}\right)\right]\right)$.

Step 7: calculate the ranking vector $\left\{P_{1}, P_{2}, \ldots, P_{n}\right\}$ of the possibility matrix $P=\left(p_{i j}\right)_{n \times n}$, where $P_{i}=1 / n(n-1)\left(\sum_{j=1}^{n} p_{i j}-1+(n / 2)\right),\{i=1,2, \ldots, n\}$.

According to the size of $P_{i}$, the candidate scheme is selected for the optimal decision; the larger the value $P_{i}$, the better the scheme.

\section{Case Analysis}

Example 2. Energy issues have always plagued mankind. Many countries and regions are committed to the development and utilization of new energy technologies. Nea-Kessani is a rural community in northern Greece. The region's economy is in a recession. The local government has decided to use clean energy and advanced manufacturing to provide new jobs to society and realize the transformation from traditional agriculture to modern industry. The locals decided to develop low-enthalpy geothermal resources, evaluated five mining methods as $x_{i}\{i=1,2, \ldots, 5\}$, and formulated the following decision attributes based on its own characteristics: $c_{1}$ is the net present value of investment, $c_{2}$ is the number of jobs created, $c_{3}$ is the resource utilization, $c_{4}$ is safety, and $c_{5}$ is social impact force. Its weight vector is $w=(0.2,0.4,0.1,0.1,0.2)$. The expert group evaluated the above five attributes with real numbers between 0 and 1 and formed a consensus evaluation opinion, giving the following evaluation matrix $H^{(i)}(i=1,2,3)$. In order to achieve scientific and reasonable decision-making effects, the local government decided to hire three industry experts $D_{i}(i=1,2,3)$ to evaluate the mining plan. In addition, weights were assigned according to their professional influence, and the weight vector is $\lambda=(0.35,0.3,0.35)$. After investigation, Pythagorean fuzzy decision matrix provided by the experts is represented in Tables 1-3.

Next, the decision-making method of this paper is used to select the best mining plan for the local area.

Step 1: since the five evaluation indicators specified by the company are all benefit-based, there is no need to standardize, that is, $\bar{H}^{(k)}=H^{(k)}=\left(\alpha_{i j}^{(k)}\right)_{n \times m}, k=1,2,3$.

Step 2: calculate the degree of support between variables $\operatorname{Sup}\left(\bar{\alpha}_{l i}^{(k)}, \bar{\alpha}_{l j}^{(k)}\right)=1-d\left(\bar{\alpha}_{l i}^{(k)}, \bar{\alpha}_{l j}^{(k)}\right), i, j=1,2, \ldots, m, l=$ $1,2, \ldots, n$, and $k=1,2,3$. Taking the evaluation matrix $H^{(1)}$ given by Expert 1 as an example, the following support matrix can be obtained:
TABle 1: Pythagorean decision matrix $H^{(1)}$.

\begin{tabular}{cccccc}
\hline & $c_{1}$ & $c_{2}$ & $c_{3}$ & $c_{4}$ & $c_{5}$ \\
\hline$x_{1}$ & $P(0.4,0.8)$ & $P(0.7,0.6)$ & $P(0.6,0.7)$ & $P(0.3,0.8)$ & $P(0.5,0.6)$ \\
$x_{2}$ & $P(0.7,0.5)$ & $P(0.9,0.2)$ & $P(0.8,0.5)$ & $P(0.3,0.6)$ & $P(0.6,0.5)$ \\
$x_{3}$ & $P(0.3,0.4)$ & $P(0.3,0.7)$ & $P(0.7,0.4)$ & $P(0.6,0.4)$ & $P(0.4,0.5)$ \\
$x_{4}$ & $P(0.6,0.6)$ & $P(0.7,0.5)$ & $P(0.7,0.2)$ & $P(0.4,0.6)$ & $P(0.3,0.7)$ \\
$x_{5}$ & $P(0.5,0.7)$ & $P(0.6,0.4)$ & $P(0.9,0.3)$ & $P(0.6,0.7)$ & $P(0.1,0.7)$ \\
\hline
\end{tabular}

TABle 2: Pythagorean decision matrix $H^{(2)}$.

\begin{tabular}{cccccc}
\hline & $c_{1}$ & $c_{2}$ & $c_{3}$ & $c_{4}$ & $c_{5}$ \\
\hline$x_{1}$ & $P(0.3,0.9)$ & $P(0.7,0.6)$ & $P(0.5,0.8)$ & $P(0.3,0.6)$ & $P(0.3,0.6)$ \\
$x_{2}$ & $P(0.7,0.4)$ & $P(0.9,0.2)$ & $P(0.8,0.1)$ & $P(0.3,0.5)$ & $P(0.3,0.5)$ \\
$x_{3}$ & $P(0.3,0.6)$ & $P(0.7,0.7)$ & $P(0.7,0.6)$ & $P(0.4,0.4)$ & $P(0.4,0.3)$ \\
$x_{4}$ & $P(0.4,0.8)$ & $P(0.7,0.5)$ & $P(0.6,0.2)$ & $P(0.4,0.7)$ & $P(0.4,0.7)$ \\
$x_{5}$ & $P(0.2,0.7)$ & $P(0.8,0.2)$ & $P(0.8,0.4)$ & $P(0.6,0.6)$ & $P(0.6,0.6)$ \\
\hline
\end{tabular}

TABle 3: Pythagorean decision matrix $H^{(3)}$.

\begin{tabular}{cccccc}
\hline & $c_{1}$ & $c_{2}$ & $c_{3}$ & $c_{4}$ & $c_{5}$ \\
\hline$x_{1}$ & $P(0.6,0.7)$ & $P(0.7,0.6)$ & $P(0.5,0.8)$ & $P(0.5,0.5)$ & $P(0.1,0.6)$ \\
$x_{2}$ & $P(0.6,0.5)$ & $P(0.9,0.2)$ & $P(0.8,0.1)$ & $P(0.3,0.5)$ & $P(0.3,0.4)$ \\
$x_{3}$ & $P(0.4,0.7)$ & $P(0.7,0.5)$ & $P(0.6,0.1)$ & $P(0.2,0.9)$ & $P(0.6,0.5)$ \\
$x_{4}$ & $P(0.2,0.9)$ & $P(0.5,0.6)$ & $P(0.6,0.2)$ & $P(0.1,0.6)$ & $P(0.4,0.7)$ \\
$x_{5}$ & $P(0.1,0.6)$ & $P(0.8,0.2)$ & $P(0.9,0.2)$ & $P(0.6,0.5)$ & $P(0.4,0.6)$ \\
\hline
\end{tabular}

$$
\begin{aligned}
& S^{(1)}(1)=\left(\begin{array}{ccccc}
1 & 0.67 & 0.80 & 0.93 & 0.72 \\
0.67 & 1 & 0.87 & 0.60 & 0.76 \\
0.80 & 0.87 & 1 & 0.73 & 0.76 \\
0.93 & 0.60 & 0.73 & 1 & 0.72 \\
0.72 & 0.76 & 0.76 & 0.72 & 1
\end{array}\right) \text {, } \\
& S^{(1)}(2)=\left(\begin{array}{ccccc}
1 & 0.68 & 0.85 & 0.60 & 0.87 \\
0.68 & 1 & 0.79 & 0.28 & 0.55 \\
0.85 & 0.79 & 1 & 0.45 & 0.72 \\
0.60 & 0.28 & 0.45 & 1 & 0.73 \\
0.87 & 0.55 & 0.72 & 0.73 & 1
\end{array}\right) \text {, } \\
& S^{(1)}(3)=\left(\begin{array}{ccccc}
1 & 0.67 & 0.60 & 0.73 & 0.84 \\
0.67 & 1 & 0.60 & 0.67 & 0.76 \\
0.60 & 0.60 & 1 & 0.87 & 0.67 \\
0.73 & 0.67 & 0.87 & 1 & 0.80 \\
0.84 & 0.76 & 0.67 & 0.80 & 1
\end{array}\right) \text {, } \\
& S^{(1)}(4)=\left(\begin{array}{ccccc}
1 & 0.87 & 0.68 & 0.80 & 0.73 \\
0.87 & 1 & 0.79 & 0.67 & 0.60 \\
0.68 & 0.79 & 1 & 0.67 & 0.55 \\
0.80 & 0.67 & 0.67 & 1 & 0.87 \\
0.73 & 0.60 & 0.55 & 0.87 & 1
\end{array}\right) \text {, } \\
& S^{(1)}(5)=\left(\begin{array}{ccccc}
1 & 0.67 & 0.44 & 0.89 & 0.76 \\
0.67 & 1 & 0.55 & 0.67 & 0.65 \\
0.44 & 0.55 & 1 & 0.55 & 0.20 \\
0.89 & 0.67 & 0.55 & 1 & 0.65 \\
0.76 & 0.65 & 0.20 & 0.65 & 1
\end{array}\right) \text {. }
\end{aligned}
$$


Step 3: calculate the support $T\left(\bar{\alpha}_{l i}^{(k)}\right)$ of the variable and the remaining variables and the support index of the variables $\eta_{l i}^{(k)}, \quad i=1,2, \ldots, m, \quad l=1,2, \ldots, n$, and $k=1,2,3$. Then, the following support matrix $T^{(k)}$ and support index matrix $\eta^{(k)}$ can be obtained:

$$
\begin{aligned}
& T^{(1)}=\left(\begin{array}{lllll}
3.12 & 2.90 & 3.16 & 2.98 & 2.96 \\
3.00 & 2.30 & 2.81 & 2.06 & 2.87 \\
2.84 & 2.70 & 2.74 & 3.07 & 3.07 \\
3.08 & 2.93 & 2.69 & 3.01 & 2.75 \\
2.76 & 2.54 & 1.74 & 2.76 & 2.26
\end{array}\right), \\
& \eta^{(1)}=\left(\begin{array}{ccccc}
0.21 & 0.39 & 0.11 & 0.10 & 0.20 \\
0.23 & 0.36 & 0.11 & 0.08 & 0.22 \\
0.20 & 0.38 & 0.10 & 0.11 & 0.22 \\
0.21 & 0.40 & 0.09 & 0.10 & 0.19 \\
0.22 & 0.41 & 0.07 & 0.11 & 0.18
\end{array}\right) \text {, } \\
& T^{(2)}=\left(\begin{array}{lllll}
2.48 & 2.47 & 2.67 & 2.71 & 2.71 \\
2.73 & 2.04 & 2.55 & 2.33 & 2.33 \\
2.60 & 1.95 & 2.34 & 2.54 & 2.33 \\
2.71 & 2.61 & 2.16 & 3.07 & 3.07 \\
2.16 & 2.64 & 2.72 & 3.08 & 3.08
\end{array}\right), \\
& \eta^{(2)}=\left(\begin{array}{lllll}
0.19 & 0.39 & 0.10 & 0.11 & 0.21 \\
0.24 & 0.35 & 0.11 & 0.10 & 0.20 \\
0.23 & 0.35 & 0.10 & 0.11 & 0.21 \\
0.20 & 0.38 & 0.08 & 0.11 & 0.23 \\
0.16 & 0.39 & 0.10 & 0.11 & 0.23
\end{array}\right), \\
& T^{(3)}=\left(\begin{array}{lllll}
2.89 & 2.76 & 2.66 & 2.67 & 2.28 \\
2.64 & 1.91 & 2.42 & 2.37 & 2.28 \\
2.63 & 2.61 & 2.11 & 1.76 & 2.83 \\
1.98 & 2.86 & 2.11 & 2.65 & 2.82 \\
2.07 & 2.44 & 1.93 & 2.72 & 2.52
\end{array}\right) \text {, } \\
& \eta^{(3)}=\left(\begin{array}{ccccc}
0.22 & 041 & 0.10 & 0.10 & 0.17 \\
0.24 & 0.34 & 0.11 & 0.11 & 0.20 \\
0.21 & 0.41 & 0.08 & 0.07 & 0.22 \\
0.15 & 0.44 & 0.08 & 0.10 & 0.22 \\
0.18 & 0.41 & 0.08 & 0.12 & 0.21
\end{array}\right) .
\end{aligned}
$$

Step 4: use the PFIWPA operator to gather the attribute values $\bar{\alpha}_{i j}^{(k)}, j=1,2, \ldots, m$, of the candidate solutions to obtain expert $D_{k}$ 's comprehensive evaluation value of the candidate solutions $x_{i}$, as shown in Table 4 .
TABLE 4: Comprehensive evaluation information.

\begin{tabular}{cccc}
\hline & $D_{1}$ & $D_{2}$ & $D_{3}$ \\
\hline$x_{1}$ & $P(0.58,0.68)$ & $P(0.54,0.71)$ & $P(0.59,0.66)$ \\
$x_{2}$ & $P(0.79,0.39)$ & $P(0.77,0.31)$ & $P(0.75,0.33)$ \\
$x_{3}$ & $P(0.43,0.56)$ & $P(0.56,0.72)$ & $P(0.60,0.57)$ \\
$x_{4}$ & $P(0.61,0.55)$ & $P(0.57,0.62)$ & $P(0.43,0.67)$ \\
$x_{5}$ & $P(0.61,0.56)$ & $P(0.70,0.45)$ & $P(0.69,0.38)$ \\
\hline
\end{tabular}

Step 5: standardize the comprehensive evaluation value of the expert's candidate program, and then, use the evidence synthesis method to fuse the data. The specific results are obtained, as shown in Table 5 .

Step 6: the comprehensive evidence information of the alternatives shows that the confidence intervals of the alternatives are $I_{1}=[0.30,0.44], \quad I_{2}=[0.57,0.75]$, $I_{3}=[0.31,0.50], I_{4}=[0.32,0.51]$, and $I_{5}=[0.46,0$. 65]. Based on this, the possibility matrix $P=\left(p_{i j}\right)_{5 \times 5}$ between candidate schemes can be obtained:

$$
P=\left(\begin{array}{ccccc}
\sim & 0 & 0 & 0 & 0 \\
1 & \sim & 1 & 1 & 1 \\
1 & 0 & \sim & 0.81 & 0.22 \\
1 & 0 & 0.19 & \sim & 0 \\
1 & 0 & 0.78 & 1 & \sim
\end{array}\right) .
$$

Step 7: from the ranking vector of the degree of possibility matrix $\{0.08,0.28,0.18,0.13,0.21\}$, the ranking of candidate solutions can be obtained as $x_{2}>x_{5}>x_{3}>x_{4}>x_{1}$.

Therefore, the best green supplier should is $x_{2}$.

\section{Method Comparison and Discussion}

Research on Pythagorean fuzzy group decision-making has just started, and there are few related documents. In this section, we compare the proposed method with the Pythagorean fuzzy TOPSIS group decision-making method [29] and the group decision-making method based on the WA operator [27]. Tables 6 and 7 show that the optimal choices given by the three group decision-making methods in Example 2 are both $x_{2}$, which shows that the group decision-making method in this paper is feasible. In-depth analysis from [27, 29] clearly shows that the differences between the candidate solutions are magnified. This is mainly based on the following reasons:

(1) The group decision-making methods proposed by $[27,29]$ assume that experts involved in decisionmaking are considered to be fully rational individuals. However, this assumption is not always true because of the complicated decision environment and finiteness of humans' cognitive abilities.

(2) The PFIWPA operator used in this paper can take into account the balance and relevance of the information when gathering decision information, 
TABLE 5: Results of evidence synthesis.

\begin{tabular}{|c|c|c|c|c|}
\hline & \multicolumn{3}{|c|}{ Standardized comprehensive evaluation information } & \multirow{2}{*}{ Comprehensive evidence information } \\
\hline & $D_{1}$ & $D_{2}$ & $D_{3}$ & \\
\hline$x_{1}$ & $P(0.22,0.33)$ & $P(0.19,0.33)$ & $P(0.23,0.32)$ & $(0.30,0.56,0.14)$ \\
\hline$x_{2}$ & $P(0.32,0.21)$ & $P(0.31,0.16)$ & $P(0.31,0.17)$ & $(0.57,0.25,0.17)$ \\
\hline$x_{3}$ & $P(0.17,0.25)$ & $P(0.20,0.34)$ & $P(0.24,0.26)$ & $(0.31,0.50,0.18)$ \\
\hline$x_{4}$ & $P(0.24,0.26)$ & $P(0.21,0.28)$ & $P(0.17,0.30)$ & $(0.32,0.49,0.19)$ \\
\hline$x_{5}$ & $P(0.24,0.26)$ & $P(0.27,0.21)$ & $P(0.28,0.18)$ & $(0.46,0.35,0.19)$ \\
\hline
\end{tabular}

TABLE 6: Calculation results of [27].

\begin{tabular}{lccc}
\hline Candidate plan & Comprehensive evaluation value & Score value & Sort result \\
\hline$x_{1}$ & $P(0.60,0.71)$ & -0.11 & 5 \\
$x_{2}$ & $P(0.78,0.22)$ & 0.56 & 1 \\
$x_{3}$ & $P(0.55,0.45)$ & 0.10 & 4 \\
$x_{4}$ & $P(0.55,0.39)$ & 0.16 & 3 \\
$x_{5}$ & $P(0.67,0.32)$ & 0.34 & 2 \\
\hline
\end{tabular}

TABLE 7: Calculation results of [29].

\begin{tabular}{|c|c|c|c|c|}
\hline Candidate plan & $D\left(x_{i}, x^{+}\right)$ & $D\left(x_{i}, x^{-}\right)$ & The relative closeness of the alternative & Sort result \\
\hline$x_{1}$ & 0.259 & 0.154 & 0.374 & 5 \\
\hline$x_{2}$ & 0.1 & 0.342 & 0.774 & 1 \\
\hline$x_{3}$ & 0.224 & 0.253 & 0.53 & 3 \\
\hline$x_{4}$ & 0.247 & 0.216 & 0.466 & 4 \\
\hline$x_{5}$ & 0.172 & 0.265 & 0.607 & 2 \\
\hline
\end{tabular}

while the group decision-making methods used in literatures [27, 29] use independent attributes by default. Moreover, the model calculation of [27, 29] is based on Definition 4. As mentioned above, this type of calculation rule has defects, which can easily distort decision information.

(3) When constructing the decision-making model, this paper uses evidence theory to fuse evaluation information among experts, which can be closer to human cognition than a collective settlement in a bounded rational environment [28].

(4) The decision method given in [27, 29] uses the PFWA operator and TOPSIS when aggregating the evaluation information of different attributes and the evaluation information between experts, respectively. This paper uses the PFIWPA operator and the evidence theory to deal with the characteristics of information aggregation at different stages. The proposed method integrates the advantages of multiple methods to comprehensively solve the problem of aggregating of group decision information.

\section{Conclusion}

Aiming at the problem of multiple attribute group decisionmaking in Pythagorean fuzzy environment, this paper uses evidence theory and the Pythagorean fuzzy interactive weighted power average operator to propose a group decision-making method. The new method uses the Pythagorean fuzzy interactive weighted power average operator to aggregate experts' attribute evaluation information and gives the comprehensive evaluation information of each expert. The operator used is better than the commonly used power average operator and not only satisfies idempotence and exchangeability but also mines attribute associations. Then, the rules of evidence synthesis are used to gather the comprehensive evaluation information of experts and give the comprehensive evidence information of the candidate program. Finally, the evidence information is used to give the best ranking of the candidate solutions. However, this method also has its shortcomings. The situation in which the attribute evaluation value is the interval Pythagorean fuzzy number and the attribute weight is unknown is not discussed. The next step will focus on the Pythagorean fuzzy group decisionmaking problem in these situations.

\section{Data Availability}

Data used to support the findings of this study are available from the corresponding author upon request.

\section{Conflicts of Interest}

The authors declare that there are no conflicts of interest.

\section{Acknowledgments}

This work was supported by the National Natural Science Foundation of China (Grant no. 11171221), the Education 
Department of Anhui Province Humanities and Social Sciences Key Planning Fund (Grant no. SK2015A563), the Natural Science Foundation of Anhui Province (Grant no. 1908085QG306), Ministry of Education Humanities and Social Sciences Research Youth Fund Project (Grant no. 18YJCZH216), and the Key Specialized Research and Development Breakthrough in Henan Province (Grant no. 202102310637).

\section{References}

[1] R. E. Bellman and L. A. Zadeh, "Decision-making in a fuzzy environment," Management Science, vol. 17, pp. 141-164, 1970.

[2] K. T. Atanassov, More Information about Intuitionistic Fuzzy Sets, North Holland Province, Amsterdam, The Netherlands, 1989.

[3] R. R. Yager, "Pythagorean membership grades in multicriteria decision making," IEEE Transactions on Fuzzy Systems, vol. 22, no. 4, pp. 958-965, 2014.

[4] T.-T. Xu, H. Zhang, and B.-Q. Li, "Pythagorean fuzzy entropy and its application in multiple-criteria decision-making," International Journal of Fuzzy Systems, vol. 22, no. 5, pp. 1552-1564, 2020.

[5] K. Rahman, "Approaches to some induced einstein geometric aggregation operators based on interval-valued Pythagorean fuzzy numbers and their application," New Mathematics and Natural Computation, vol. 16, no. 2, pp. 211-230, 2020.

[6] T. K. Paul, M. Pal, and C. Jana, "Multi-attribute decision making method using advanced Pythagorean fuzzy weighted geometric operator and their applications for real estate company selection," Heliyon, vol. 7, pp. 1-9, 2021.

[7] H. Garg, "Sine trigonometric operational laws and its based Pythagorean fuzzy aggregation operators for group decisionmaking process," Artificial Intelligence Review, vol. 54, no. 6 , pp. 4421-4447, 2021.

[8] Q. Zhang, J. Hu, J. Feng, A. Liu, and Y. Li, "New similarity measures of Pythagorean fuzzy sets and their applications," IEEE Access, vol. 7, pp. 138192-138202, 2019.

[9] S.-P. Wan, Z. Jin, and J.-Y. Dong, "A new order relation for Pythagorean fuzzy numbers and application to multi-attribute group decision making," Knowledge and Information Systems, vol. 62, no. 2, pp. 751-785, 2020.

[10] P. A. Ejegwa, "Generalized triparametric correlation coefficient for Pythagorean fuzzy sets with application to MCDM problems," Granular Computing, vol. 6, no. 3, pp. 557-566, 2021.

[11] N. X. Thao, "A new correlation coefficient of the Pythagorean fuzzy sets and its applications," Soft Computing, vol. 24, no. 13, pp. 9467-9478, 2020.

[12] V. Chinnadurai and A. Arulselvam, "Q-Pythagorean fuzzy soft expert set and its application in multi-criteria decision making process," Journal of Physics: Conference Series, vol. 1850, pp. 1-11, 2021.

[13] L. Zhang, L. Zhou, and K. Yang, "Consistency analysis and priorities deriving for pythagorean fuzzy preference relation in the "computing in memory" IEEE Access, vol. 8, pp. 156972-156985, 2020.

[14] Y. Liu, J. Liu, and Y. Qin, "Pythagorean fuzzy linguistic Muirhead mean operators and their applications to multiattribute decision-making," International Journal of Intelligent Systems, vol. 35, no. 2, pp. 300-332, 2020.
[15] L. Wang, H. Garg, and N. Li, "Pythagorean fuzzy interactive Hamacher power aggregation operators for assessment of express service quality with entropy weight," Soft Computing, vol. 25, no. 2, pp. 973-993, 2021.

[16] C. Ji, R. Zhang, and J. Wang, "Probabilistic dual-hesitant pythagorean fuzzy sets and their application in multi-attribute group decision-making," Cognitive Computation, vol. 13, no. 4, pp. 919-935, 2021.

[17] S.-P. Wan, Z. Jin, and J.-Y. Dong, "Pythagorean fuzzy mathematical programming method for multi-attribute group decision making with Pythagorean fuzzy truth degrees," Knowledge and Information Systems, vol. 55, no. 2, pp. 437-466, 2018.

[18] M. Akram, S. Alsulami, and K. Zahid, "A hybrid method for complex Pythagorean fuzzy decision making," Mathematical Problems in Engineering, vol. 2021, Article ID 9915432, 23 pages, 2021.

[19] X. Ma M, A. K. Zahid, and C. R. Alcantud, "Group decisionmaking framework using complex Pythagorean fuzzy information," Neural Computing \& Applications, vol. 33, pp. 2085-2105, 2019.

[20] S.-P. Wan, S.-Q. Li, and J.-Y. Dong, "A three-phase method for Pythagorean fuzzy multi-attribute group decision making and application to haze management," Computers \& Industrial Engineering, vol. 123, pp. 348-363, 2018.

[21] M. Akram, X. Peng, and A. Sattar, "Multi-criteria decisionmaking model using complex Pythagorean fuzzy yager aggregation operators," Arabian Journal for Science and Engineering, vol. 46, no. 2, pp. 1691-1717, 2021.

[22] R. R. Yager, “The power average operator," IEEE Transactions on Systems, Man, and Cybernetics-Part A: Systems and Humans, vol. 31, pp. 724-731, 2011.

[23] W. Qiu and J. Qiu, "Probabilistic hesitant triangular fuzzy power aggregation operator and its application," IOP Conference Series: Materials Science and Engineering, vol. 768, pp. 1-9, 2020.

[24] Y. He, H. Chen, L. Zhou, J. Liu, and Z. Tao, "Intuitionistic fuzzy geometric interaction averaging operators and their application to multi-criteria decision making," Information Sciences, vol. 259, pp. 142-159, 2014.

[25] G. Shafer, The Mathematical Theory of Evidence, Princeton University Press, Princeton, NJ, USA, 1976.

[26] A. P. Dempster, "Upper and lower probabilities induced by a multivalued mapping," The Annals of Mathematical Statistics, vol. 38, no. 2, pp. 325-339, 1967.

[27] X. Zhang, "A novel approach based on similarity measure for pythagorean fuzzy multiple criteria group decision making," International Journal of Intelligent Systems, vol. 31, no. 6, pp. 593-611, 2016.

[28] R. R. Yager, "Belief structures, weight generating functions and decision-making," Fuzzy Optimization and Decision Making, vol. 16, no. 1, pp. 1-21, 2017.

[29] A. Muhammad, D. Wieslaw, and I. Farwa, "Group decisionmaking based on Pythagorean fuzzy TOPSIS method," International journal of entelligent systems, vol. 34, pp. 1455$1475,2019$.

[30] C. Huang, M. Lin, and Z. Xu, "Pythagorean fuzzy MULTIMOORA method based on distance measure and score function: its application in multicriteria decision making process," Knowledge and Information Systems, vol. 62, no. 11, pp. 4373-4406, 2020. 